\title{
Highly Sensitive Capacitive Sensor Based on Injection Locked Oscillators with ppm Sensing Resolution
}

\author{
Meissa Babay ${ }^{\# 1}$, Clement Hallepee ${ }^{\#}$, Claire Dalmay ${ }^{\#}$ Bruno Barelaud ${ }^{\#}$, Emre Can Durmaz , \\ Canan Baristiran Kaynak*, Mehmet Kaynak* ${ }^{*}$, David Cordeau ${ }^{\$ 2}$, Arnaud Pothier ${ }^{\# 3}$ \\ \# XLIM- CNRS- University of Limoges, France \\ ${ }^{\$}$ XLIM - CNRS- University of Poitiers, France \\ * IHP - Leibniz-Institut für innovative Mikroelektronik, Frankfurt (Oder), Germany \\ 1'meissa.babay@xlim.fr, ${ }^{2}$ david.cordeau@xlim.fr, ${ }^{3}$ arnaud.pothier@xlim.fr
}

\begin{abstract}
This paper presents the design and the implementation of an ultra-sensitive capacitive sensor based on an injection-locked oscillator architecture able to achieve ppm sensing resolution. The proposed RF IC chip, implemented on a BiCMOS SiGe $0.25 \mu \mathrm{m}$ technology, is able to detect capacitance changes in the range of few attoF, induced by dielectric disturbance occurring on on-chip integrated sensing capacitors. Indeed, this paper demonstrates that, once locked on a $4.693 \mathrm{GHz}$ and $\mathbf{- 5 0} \mathrm{dBm}$ injection signal, the proposed sensing system can efficiently detect differences of free running frequencies as low as $10 \mathrm{kHz}$ between two oscillators. Considering a differential measurement approach with two injection locked oscillators (one used as a sensor, the other as a reference), a 3 attoF difference between both $\mathrm{LC}$ tank sensing capacitors might be detectable. This results, in the present case, in a 5 ppm sensing sensitivity.

Keywords-RF sensors, injection locked oscillator, BiCMOS, dielectric characterization
\end{abstract}

\section{INTRODUCTION}

Dielectric capacitive sensors currently find widespread use in various domains such as medical diagnostics, food safety control [1], air pollution [2] or toxic gas detection [3], thanks to the high level of sensitivity that such sensors can reach [4-6]. Research activities are multiplying to develop sensors with small size, low cost and low energy consumption. Recent works have shown the potential of Integrated Circuits (ICs) and CMOS technology for the miniaturization and the integration of RF characterization devices. In addition, several works have demonstrated how the sensitivity of such devices can be significantly enhanced by the use of oscillator-based sensors. In this study, the proposed sensing method consists in measuring a capacitance change due to a dielectric disturbance on the sensor, which leads to an output frequency change of the oscillator-based device. This sensing approach can be applied in different types of analysis depending on the targeted application (moisture control, gas detection, bio-detection....) and on the sensitive material used. For example, in the biomedical domain, capacitive bio-sensing systems are wellknown to have great potential applications for single cell analysis based on label-free and non-destructive detection [7]. Besides, it is also possible to monitor basic environmental parameters such as the relative humidity [8] with such dielectric capacitive sensors. In addition, the development of emerging IoT gas sensing systems presents a great interest as it allows for precise monitoring of different types of environment. Capabilities of measuring the concentration of greenhouse gas such as alcohol vapor, aldehydes, organic acids, and aromatic hydrocarbons, find various applications like air quality control with environmental issues: in the context of the fight against global warming, such highly sensitive sensors can be strategic [9].

In this context, this paper presents a new differential architecture of an ultra-sensitive dielectric capacitive sensor based on injection-locked oscillators (ILOs) integrated on a BiCMOS SiGe $0.25 \mu \mathrm{m}$ process. The proposed architecture has the potential to reach a very high level of sensitivity which makes it to be used as a versatile tool. The paper is organized as follows. Section II describes the proposed sensing system and reminds the principle of an ILO. Section III presents the circuit design and the experimental results. Finally, a conclusion is given in section IV.

\section{SENSOR ARCHITECTURE}

A. Proposed sensing system

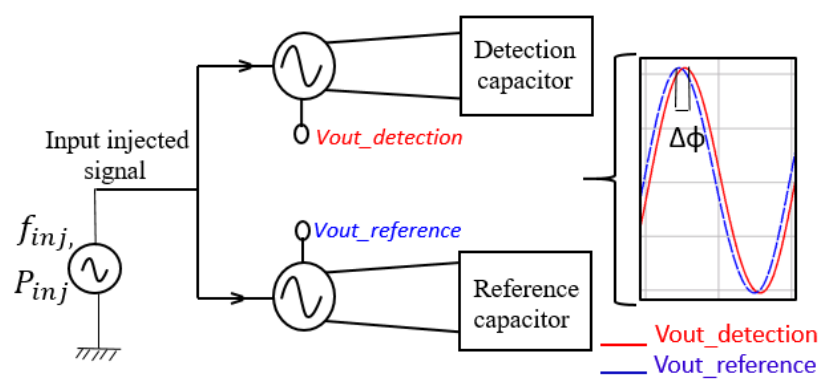

Fig. 1. Sensing system schematic diagram.

The objective of the proposed work is to implement, on a silicon chip, an ultrasensitive capacitive sensor architecture reaching a sensitivity in the order of the ppm. Generally, fully integrated oscillators suffer from one major constraint that may limit the performances of the sensing system: they can be very sensitive to environmental perturbations (temperature, noise, electromagnetic interferences...) which can cause a significant timing jitter at the output of the oscillator. For the targeted objective in this work, strong stability and very low phase noise 
of the oscillator is compulsory required in order to efficiently detect any frequency shifts induced by the event to monitor, even more in the case of very low sensing capacitance changes (ppm resolution is targeted). To overcome this issue and achieve high sensitivity sensing, the implementation of an injection locked oscillator can be a relevant approach to limit the phase noise near the carrier and reach a strong frequency stability.

In these conditions, the proposed system aims to detect any minor dielectric disturbances in the vicinity of the detection capacitor by measuring the induced phase difference between two identical LC oscillators output signals locked on the same injection signal (Fig.1); considering that only one (Detector) will be exposed, whereas the other (Reference) will not be affected by the dielectric perturbation. Hence, when a disturbance occurs and only affects the detection capacitor, it induces a small sensing capacitance change and so the freerunning frequency of the corresponding oscillator (Detector) is brought to vary in similar proportions with respect to the one of the reference. Under injection, this frequency difference will lead to a phase shift between the output signals of the two oscillators. As illustrated in Fig. 1, this differential measurement approach should ensure better sensing accuracy by self-correcting unwanted frequency deviations potentially induced by environmental perturbations. Under these conditions, the two oscillators free-running frequencies should vary in the same amount depending on the environment but only the variations that rely on the phenomenon to monitor will be characterized.

Fig. 2 describes the fundamental blocks of the proposed Injection-locked detection system. At the input, the singleended injected signal is converted into a differential signal using an external off-chip RF balun. This differential signal is subsequently applied to an injection circuit associated to the LC oscillator. The differential output signal from the sensor is then buffered. Furthermore, a differential amplifier is implemented at the output of the oscillator followed by a buffer stage in order to combine the differential output into a single $50 \Omega$-matched output.

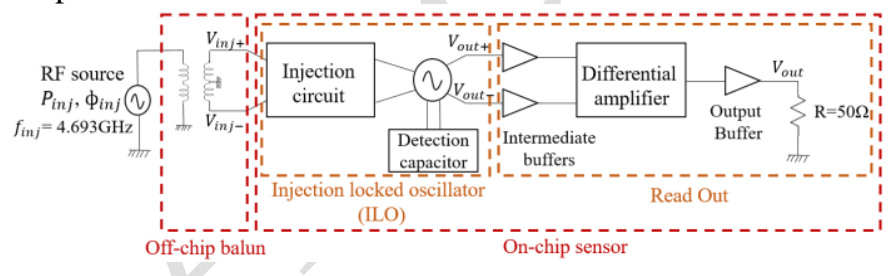

Fig. 2. Detection oscillator schematic implementation.

\section{$B$. Injection locking theory}

The injection-locking phenomena was first introduced by Adler [10] for weak injection level, and after that by Razavi [11] for high injection level. The principle of the injectionlocking is based on the fact that a master oscillator, modeled as a spectrally pure source, imposes, under certain conditions, its frequency and its phase noise close to the carrier on a slave oscillator. In these conditions, a phase shift between the output signals of the two oscillators is generated. This phase difference can be controlled by tuning the free-running frequency of the slave oscillator $[10,11]$. Actually, the behavior of an injectionlocked oscillator can be modelled using the Adler's equation for a weak injection level (1):

$$
\omega_{\operatorname{det} F R}-\omega_{\text {inj }}=\frac{\omega_{\operatorname{det} F R}}{2 Q} \cdot \frac{A_{\text {inj }}}{A_{\text {osc }}} \cdot \sin \left(\phi_{\text {det }}-\phi_{\text {inj }}\right)
$$

Where $\omega_{\text {inj }}, \phi_{\text {inj }}$ and $\mathrm{A}_{\text {inj }}$ are respectively the pulsation, the phase and the amplitude of the injected signal, $\omega_{\operatorname{det} F R}$ the free running pulsation and $\phi_{\text {det }}$ and $\mathrm{A}_{\text {osc }}$ the phase and the amplitude of the output signal of the injection-locked slave oscillator. For our purpose, capacitance variations are expected to be in the order of few hundreds to few tens of attofarads. In order to properly detect such minor capacitive variations, it is necessary to work on low injection mode (Ainj<<Aosc) to ensure a generated phase shift that might linearly vary between $\left[-30^{\circ}\right.$, $\left.+30^{\circ}\right]$.

\section{CIRCUIT DESIGN AND MEASUREMENT RESULTS}

\section{A. Circuit Design}

As mentioned before, the proposed circuit is composed of one pair of differential injection-locked oscillators (Detection \& Reference). Fig. 3 shows the schematic of the detection injection-locked oscillator. The differential LC oscillator is based on a NMOS cross-coupled architecture used here to provide the negative resistance to compensate the losses associated with the resonator. The implemented resonator is made of a differential inductor $\left(\mathrm{L}_{\text {diff }}\right)$, a high Q Metal insulator Metal capacitor $\left(\mathrm{C}_{\mathrm{MIM}}\right)$ and a sensing capacitor $\left(\mathrm{C}_{\text {Sens }}\right)$, all associated in parallel. The injected signal in the differential branches is applied through the gates of $M_{3,4}$ NMOS transistors. Output signals from $M_{1,2}$ drains $\left(\mathrm{V}_{\text {out }} \& \mathrm{~V}_{\text {out }}\right)$ are then buffered and transferred to the differential amplifier to allow sensor oscillation measurements with a high-speed oscilloscope.

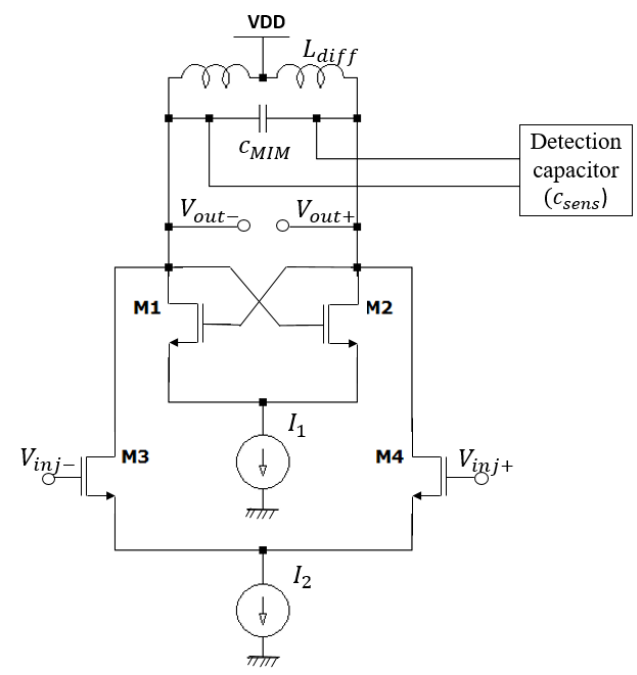

Fig. 3. Differential injection-locked oscillator schematic

B. Implementation and Experimental Results 
The proposed circuit was implemented in the SG25H4 SiGe:C BiCMOS $0.25 \mu \mathrm{m}$ technology of IHP. A chip photograph with a close view on the detection oscillator is shown in Fig.4. The chip area is $1035^{*} 1800 \mu \mathrm{m}^{2}$ including all the pads. Finally, to allow easier measurements, the chip is mounted on a PCB (Fig.5).

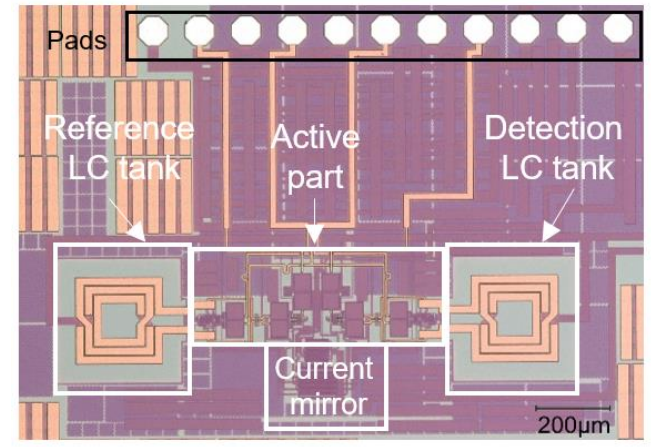

Fig. 4. Photograph of the detection system.

Fig. 5 shows the measurement setup used for evaluating the device performances. This measurement bench is composed of an external low phase noise RF source (Rohde \& Schwarz SMF100A) connected to an RF balun (Focus Microwaves BUN-750-IN). Differential outputs of this balun are mounted with circulators to limit the effects of potential signal reflections from the PCB and thus to avoid couplings between both injection locked oscillators. At the PCB outputs, both ILO output signals are directed to a coupler that allows simultaneously monitoring the sensing and reference signals on a spectrum analyzer (Rohde \& Schwarz FSV) and on an highspeed oscilloscope (Lecroy SDA 816 Zi-A 16 GHz 40 GS/s). Furthermore, batteries are used as supply voltages to avoid external parasitic signals.

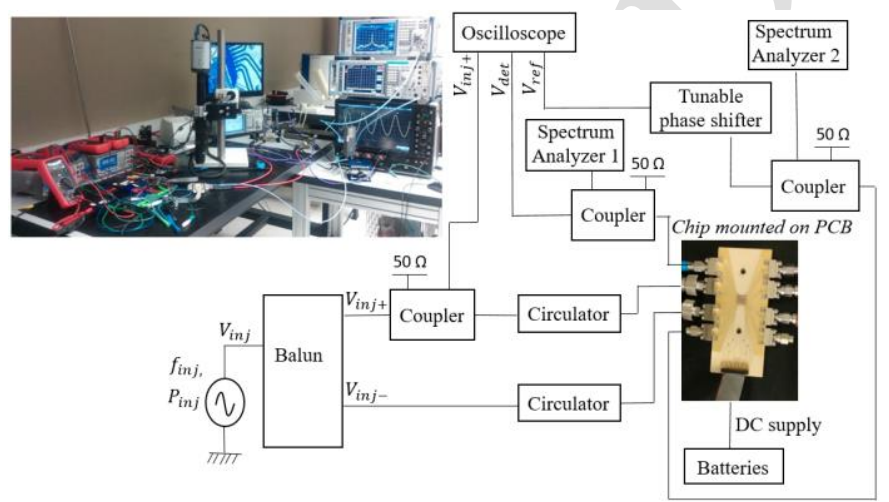

Fig. 5. Measurement setup.

In these conditions, the measurement results show that the circuit operates at a free running frequency $f_{\text {det } F R}=4.693 \mathrm{GHz}$, when no injection signal is applied. In this case, Fig. 6 (a) shows the output spectrum of either the reference or the sensing oscillator (without any disturbances) on $50 \Omega$ load. Fig.6(b) shows the output spectrum of the same oscillator locked by injection on $50 \Omega$ load when the injected frequency $f_{\text {inj }}$ is the same as the free running frequency $f_{\text {det } F R}$. As shown in
Fig. 6 (b), the phase noise near the carrier is significantly reduced compared to the case when no injection is applied. In these conditions, the proposed circuit can be more appropriated to efficiently measure sub femtofarads capacitance variations and to detect very small dielectric disturbances on the sensing capacitor.

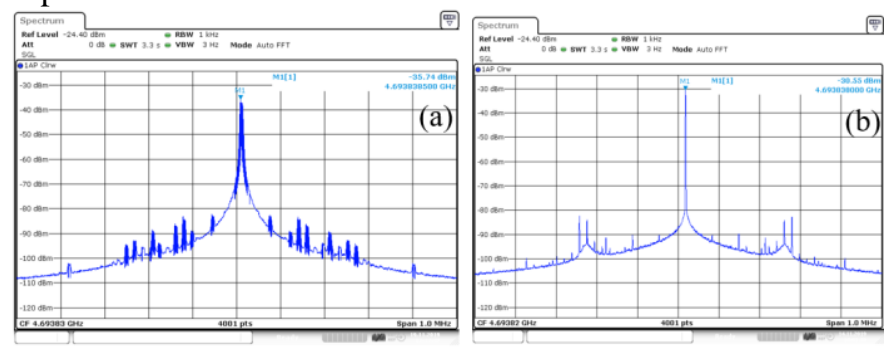

Fig. 6. Output spectrum of the oscillator in respectively (a) free-running mode and (b) locked by injection.

Fig. 7 shows the plot of the measured phase shift between the output signal of the detection oscillator with a free running frequency $f_{\text {det } F R}$ and the master signal set to a frequency $f_{i n j}$. As shown on this figure, when $f_{i n j}=f_{\operatorname{det} F R}$, the resulting phase shift $\phi_{\text {det }}-\phi_{\text {inj }}$ is null. Now thanks to external bias, the capacitance brought back by $M_{1,2}$ transistors in parallel with $\mathrm{C}_{\text {Sens }}$ can be slightly tuned. It allows to artificially change $f_{\operatorname{det} F R}$ thus resulting in a phase shift variation whose value can be tailored by adjusting the amplitude of the injected signal (1). Hence, with the proposed circuit design and by reducing the injection level in the order of $-50 \mathrm{dBm}$, the measurement results, depicted in Fig.7, show the system capability to accurately monitor a low frequency variation ranging from -100 $\mathrm{kHz}$ to $+100 \mathrm{kHz}$ around $4.693 \mathrm{GHz}$. Indeed, in such injection conditions, it still leads to a phase shift ranging between $-30^{\circ}$ and $+30^{\circ}$. Therefore, considering a minimum $3^{\circ}$ detectable phase difference, one can see that the minimal achievable measurement resolution is close to $10 \mathrm{kHz}$ frequency shift for a single ILO sensor.

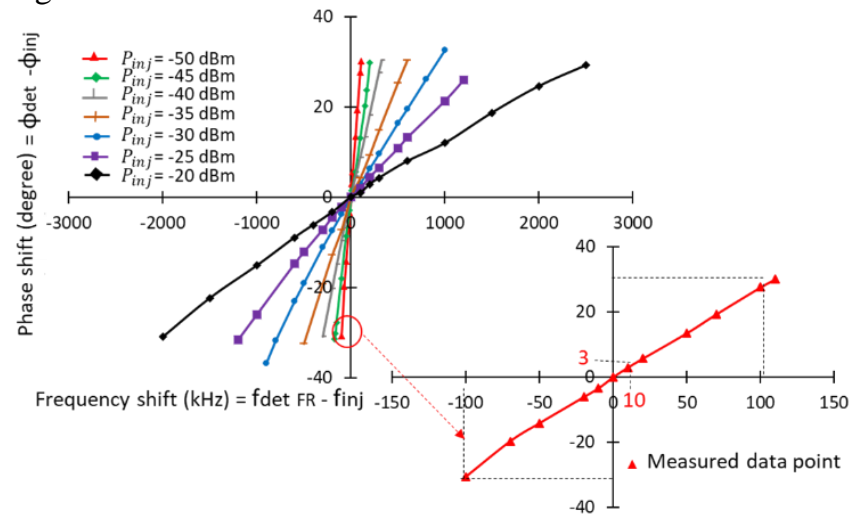

Fig. 7. Single ILO measured phase shift versus the free running frequency variation related to the injection signal one.

Now considering a differential measurement between the detector and the reference ILOs, Fig. 8 shows the resulting phase shift measured between the output signals of both injection-locked oscillators with the same injection external RF 
source $\left(f_{\text {inj } j}=f_{\operatorname{det} F R}=f_{\operatorname{ref} F R}=4.693 \mathrm{GHz}\right)$. Once lowering the injected signal power down to $-50 \mathrm{dBm}$ to reach the highest sensibility of the detection system, the free running frequency $f_{\operatorname{det} F R}$ of the detector oscillator has been artificially tuned; letting the reference oscillator's free running frequency unaffected. The resulting phase shift $\phi_{\text {det }}-\phi_{\text {ref }}$ was measured with the oscilloscope of the experimental set-up (Fig. 8, 9 \& 10) whereas, turning off the injection signal, both oscillators free running frequencies have been also recorded, thanks to the two spectrum analyzers synchronized with a common $10 \mathrm{MHz}$ clock signal. As shown in Fig.8, a very good agreement is obtained between measured sensing capability and expected performances simulated with the software Cadence. The measured phase shift is linked to the frequency variation between the detection and the reference oscillators through an almost linear relationship. Considering an implemented $1.7 \mathrm{nH}$ inductor with a $\mathrm{Q}=20$ at $4.693 \mathrm{GHz}$, Cadence simulations predict that the frequency shift $f_{\operatorname{det} F R}-f_{\text {ref } F R}$ is due to a capacitance variation ranging from -31 to +31 attoF (Fig.8).

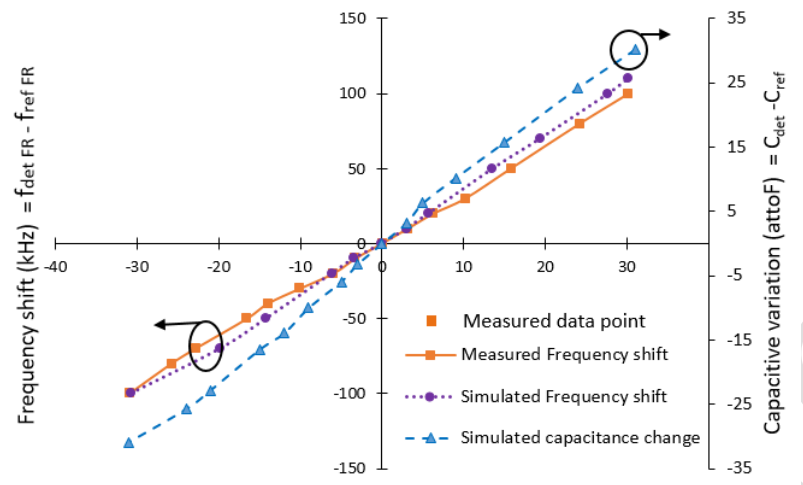

Phase shift (degree) $=\phi$ det $-\phi r e f$

Fig. 8. Differential measurement of the free running frequency change between detector and reference ILOs according to the measured phase shift between both output signals.

These simulation results can be considered to calibrate the sensor and allow, from a given phase shift, to estimate the associated sensing capacitance variation. Hence, a $3^{\circ}$ accuracy on the measured phase shift might translate in a nearly 3 attoF sensing capacitor resolution. This corresponds to an accuracy better than $5 \mathrm{ppm}$ considering that the value of the overall LC tank capacitor is close to $670 \mathrm{fF}$ in the present design.

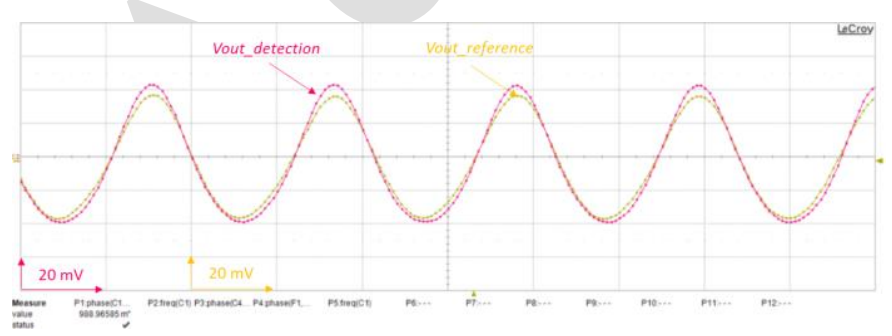

Fig. 9. Output waveform of the detection system for $f_{i n j}=f_{\operatorname{det} F R}=f_{\text {ref } F R}$, once residual phase shift is compensated.

Fig. $9 \& 10$ show the output waveforms of the two oscillators (detection and reference) under a $-50 \mathrm{dBm}$ injection power level
$\left(P_{\text {inj }}\right)$, set at $4.693 \mathrm{GHz}$. In Fig. 9, without any perturbation on the detection oscillator and once the residual phase shift (due to non-perfect symmetry of interconnections in the test bench between ILO outputs and oscilloscope (effect of cables, couplers, connectors...)) has been cancelled using a tunable phase shifter, a phase shift $\phi_{\text {det }}-\phi_{\text {ref }}=0^{\circ}$ is well obtained. Then, when a disturbance on the detection oscillator occurs, resulting in a $40 \mathrm{kHz}$ free running frequency shift, a $-13.17^{\circ}$ phase shift can be measured as shown in Fig.10.

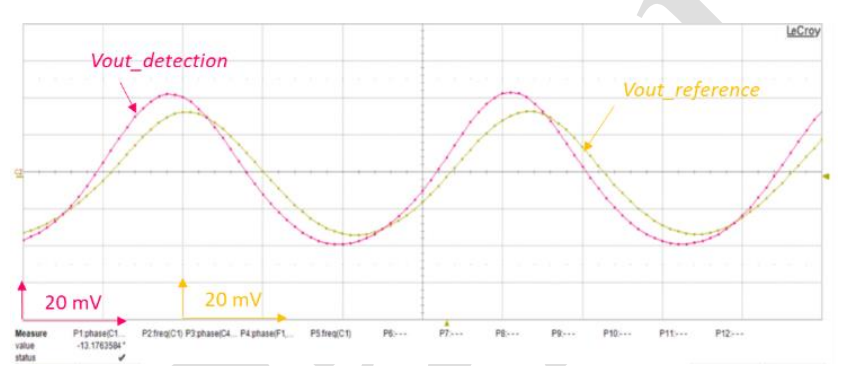

Fig. 10. Output waveforms of the detection system when $f_{\operatorname{det} F R}=f_{i n j}-40 \mathrm{kHz}$.

\section{CONCLUSION}

A high sensitivity sensing IC based on two injection- locked oscillators operating at $4.693 \mathrm{GHz}$ and implemented on a BiCMOS SiGe $0.25 \mu \mathrm{m}$ process is presented. Preliminary measurement results demonstrate the effectiveness of the sensing principle and show that such an architecture is particularly suitable for sub-femtofarad capacitance variation detection with a relative accuracy in the ppm range. Such detection level are especially required for future lab-on-chip development for biomedical diagnosis purpose.

\section{ACKNOWLEDGMENT}

This project has received funding from the European Union's Horizon 2020 research and innovation programme under grant agreement No 737164 .

\section{REFERENCES}

[1] Ee Lim Tan et al, "A Wireless, Passive Sensor for Quantifying Packaged Food Quality”, Sensors 2007

[2] Hygien Institute, Chinese Academy of Medical Sciences, "Method for detecting poisonous substance in air", China, 1974,2 (Chinese).

[3] Shwetha H.R., Rudraswamy S. B, "A Survey on Capacitive based CO2 Gas Sensor", International Journal of Engineering \& Technology, 2018.

[4] Sung Pil Lee,"Electrodes for Semiconductor Gas Sensors", J.Sensors, $2017,17,683$.

[5] Wei-Chien Lin et al, "Sensitivity improvement for CMOS-MEMS capacitive pressure sensor using double deformable diaphragms with trenches", IEEE Transducers, Kaohsiung, Taiwan, June 18-22, 2017

[6] Sih-Chieh Chen et al, "Vertically integrated CMOS-MEMS capacitive humidity sensor and a resistive temperature detector for environment application”, IEEE transducers, kaohsiung, taiwan, june 18-22, 2017

[7] J.-C. Chien et al, "Oscillator-Based Reactance Sensors With Injection Locking for High-Throughput Flow Cytometry using microwave dielectric spectroscopy", IEEE Journal of Solid-State Circuits, vol. 51, no. 2, Feb 2016

[8] Cheng-Long Zhao et al, "Enhanced performance of a CMOS interdigital capacitive humidity sensor by graphene oxide", IEEE Transducers, Beijing, China, June 5-9, 2011.

[9] C. Hagleitner et al, "CMOS single-chip gas detection system comprising capacitive, calorimetric and mass-sensitive microsensors", IEEE J. SolidSt. Circ. 2002, 37 
Paper presented during the IMS 2020 conference: Los Angeles 2-4 ${ }^{\text {th }}$ August 2020

[10] R. Adler et al, "A study of locking phenomena in oscillators", Proc. IRE, vol. 34, pp. 351-357, Jun. 1946

[11] B. Razavi et al, "A study of injection locking and pulling in oscillators," IEEE J. Solid-State Circuits, vol. 39, no. 9, pp. 1415-1424, Sep. 2004. 